\title{
ENUBET: high precision neutrino flux measurements in conventional neutrino beams
}

\section{F. Pupilli*}

INFN Sezione di Padova, via Marzolo 8 - Padova, Italy

E-mail: fabio.pupilliepd.infn.it

G. Ballerini ${ }^{a, b}$, A. Berra ${ }^{a, b}$, R. Boanta ${ }^{b, h}$, M. Bonesini $^{b}$, C. Brizzolari ${ }^{a, b}$, G. Brunetti $^{j}$,

M. Calviani ${ }^{m}$, S. Carturan ${ }^{t}$, M. G. Catanesi ${ }^{l}$, S. Cecchini ${ }^{c}$, F. Cindolo ${ }^{c}$, A. Coffani ${ }^{b, h}$,

G. Collazuol ${ }^{k, j}$, E. Conti ${ }^{j}$, F. Dal Corso ${ }^{j}$, G. De Rosa ${ }^{p, q}$, A. Gola ${ }^{o}$, R. A. Intonti ${ }^{l}$,

C. Jollet ${ }^{d}$, Y. Kudenko ${ }^{r}$, M. Laveder ${ }^{k, j}$, A. Longhin ${ }^{j}$, P.F. Loverre ${ }^{n, f}$, L. Ludovici ${ }^{f}$,

L. Magaletti ${ }^{l}$, G. Mandrioli ${ }^{c}$, A. Margotti ${ }^{c}$, V. Mascagna ${ }^{a, b}$, N. Mauri ${ }^{c}$, A. Meregaglia ${ }^{s}$,

M. Mezzetto ${ }^{j}$, M. Nessi ${ }^{m}$, A. Paoloni ${ }^{e}$, M. Pari ${ }^{k, j}$, G. Paternoster ${ }^{o}$, L. Patrizii ${ }^{c}$,

C. Piemonte ${ }^{o}$, M. Pozzato ${ }^{c}$, M. Prest ${ }^{a, b}$, E. Radicioni ${ }^{l}$, C. Riccio ${ }^{p, q}$, A. C. Ruggieri ${ }^{p}$,

M. Soldani ${ }^{a, b}$, G. Sirri ${ }^{c}$, M. Tenti $^{c}, g$, F. Terranova ${ }^{a, b}$, E. Vallazza ${ }^{i}$, M. Vesco $^{t}$,

L. Votano ${ }^{e}$, E. Wildner ${ }^{m}$

${ }^{a}$ Phys. Dep., Università degli studi dell'Insubria, via Valeggio 11, Como

${ }^{b}$ INFN, Sezione di Milano-Bicocca, piazza della Scienza 3, Milano, Italy

${ }^{c}$ INFN, Sezione di Bologna, viale Berti-Pichat 6/2, Bologna, Italy

${ }^{d}$ IPHC, Université de Strasbourg, CNRS/IN2P3, Strasbourg, France

e INFN, Laboratori Nazionali di Frascati, via Fermi 40, Frascati (Rome), Italy

${ }^{f}$ INFN, Sezione di Roma 1, piazzale A. Moro 2, Rome, Italy

$g$ Phys. Dep. Università di Bologna, viale Berti-Pichat 6/2, Bologna, Italy

${ }^{h}$ Phys. Dep. Università di Milano-Bicocca, piazza della Scienza 3, Milano, Italy

${ }^{i}$ INFN Sezione di Trieste, via Valerio, 2 - Trieste, Italy

${ }^{j}$ INFN Sezione di Padova, via Marzolo, 8 - Padova, Italy

${ }^{k}$ Phys. Dep. Università di Padova, via Marzolo, 8 - Padova, Italy

${ }^{l}$ INFN Sezione di Bari, via Amendola, 173 - Bari, Italy

${ }^{m}$ CERN, Geneva, Switzerland

${ }^{n}$ Phys. Dep. Università La Sapienza, piazzale A. Moro 2, Rome, Italy

${ }^{o}$ Fondazione Bruno Kessler (FBK) and INFN TIFPA, Trento, Italy

${ }^{p}$ INFN, Sezione di Napoli, via Cinthia, 80126, Napoli, Italy

${ }^{q}$ Phys. Dep. Università degli Studi di Napoli Federico II, via Cinthia, 80126, Napoli

${ }^{r}$ Institute of Nuclear Research of the Russian Academy of Science, Moscow, Russia

${ }^{s}$ CENBG, Université de Bordeaux, CNRS/IN2P3, 33175 Gradignan, France

${ }^{t}$ INFN Laboratori Nazionali di Legnaro, Viale dell'Università, 2 - Legnaro (PD), Italy 
The ENUBET project aims at demonstrating that the systematics in neutrino fluxes from conventional beams can be reduced to $1 \%$ by monitoring positrons from $\mathrm{K}_{e 3}$ decays in an instrumented decay tunnel, thus allowing a precise measurement of the $v_{e}$ (and $\bar{v}_{e}$ ) cross section. This contribution will report the results achieved in the first year of activities. The expected neutrino fluxes at an hypothetical far detector have been estimated. Progress have been made in the design of the hadron beamline and a complete simulation of the positron tagger allowed to determine its performance in terms of signal selection efficiency and purity. A deep R\&D program has been pursued to explore different technical solution for the tagger instrumentation. Test beam exposures of detector prototypes have been performed to assess their response to pions, electrons and muons and to validate the Monte Carlo simulation.

The 19th International Workshop on Neutrinos from Accelerators-NUFACT2017

25-30 September, 2017

Uppsala University, Uppsala, Sweden

* Speaker. 


\section{Monitored neutrino beams: the ENUBET approach}

In conventional beamlines the decay tunnel is a passive region and the knowledge of the neutrino flux relies on a complete simulation of the production beamline. Despite the use of hadroproduction data from dedicated experiments and of ancillary measurements (proton intensity, horn currents, beam-target misalignment etc.), the precision on flux prediction is usually limited to 5$10 \%$. This represents the main limiting factor for precise measurements of neutrino cross sections. In particular $v_{e}$ and $\bar{v}_{e}$ cross sections are poorly known in spite of their relevance for precision oscillation physics in the next generation of long-baseline experiments.

The reduction of flux-related systematics can be achieved by directly monitoring leptons produced in the decay tunnel together with neutrinos. If the momentum of secondary hadrons is of the order of few $\mathrm{GeV}$ and the tunnel is sufficiently short $(\sim 50 \mathrm{~m})$, the decay in flight of muons from secondary pions is suppressed and the only source of $v_{e}\left(\bar{v}_{e}\right)$ in the beamline is the $\mathrm{K}_{e 3}$ decay of charged kaons: $K^{+(-)} \rightarrow \pi^{0} e^{+(-)} v_{e}\left(\bar{v}_{e}\right)$. The measurement of emitted large angle positrons (electrons) in an instrumented decay tunnel allows a direct estimate of the $v_{e}\left(\bar{v}_{e}\right)$ flux not affected by beam related systematics arising from the number of protons on target (PoT), the hadro-production cross sections and the secondary meson focusing efficiency of the beamline. By this approach a reduction of the uncertainty on the $v$ flux normalization down to $1 \%$ can be achieved [1].

The ENUBET (Enhanced NeUtrino BEams from kaon Tagging) project is funded from June 2016 to May 2021 by the European Research Council (ERC Consolidator Grant, PI A. Longhin, host institution INFN) to demonstrate the technical feasibility and the physics performance of monitored neutrino beams. The proposed approach could be exploited by new experiments aiming at precise $(\mathscr{O}(1 \%))$ neutrino cross-section measurements and also in searches for sterile- $v$ if current anomalies are confirmed. The project would also pave the way for a "time tagged $v$ beam", where the $v_{e}$ at the detector is time-correlated with the produced $e^{+}$in the decay tunnel.

The ENUBET baseline design [2], sketched in Fig. 1, foresees a longitudinally segmented shashlik calorimeter instrumenting the decay tunnel walls (positron tagger), as a cost-effective solution to efficiently separate $\mathrm{K}_{e 3}$ positrons from muons and charged pions produced in other kaon decay modes. It is based on small units called Ultra Compact Modules (UCM). Rings of plastic scintillator doublets in the innermost region are used to reject the photon background from $\pi^{0}$ decays. The focusing and transfer line is designed to select secondary hadrons from the interactions of protons on target with a momentum of $8.5 \pm 20 \% \mathrm{GeV} / \mathrm{c}$.

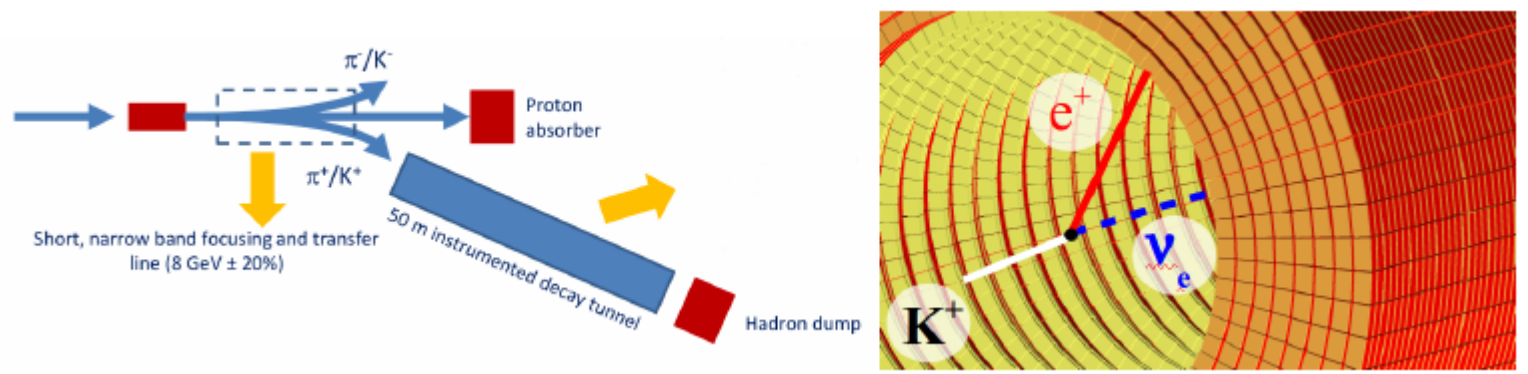

Figure 1: (left) Scheme of the complete ENUBET beamline. (right) The positron tagger, with arrays of calorimeter modules (UCM, in orange) and the photon veto pads (in yellow). 
The relatively high momentum of secondary particles is tuned to reduce the fraction of kaons decaying in the transfer line and to allow an efficient positron tagging with calorimetric techniques. Furthermore the resulting neutrino energy spectrum covers the region of interest for future long baseline experiments.

Assuming the parameters in [1] and a horn-based secondary focusing (see next section), a $500 \mathrm{t}$ liquid argon detector with a transverse size of $6 \times 6 \mathrm{~m}^{2}$ located $50 \mathrm{~m}$ after the beam dump of the ENUBET beamline would accumulate $10^{4} v_{e}^{C C}$ interactions (1\% stat. error on $\sigma\left(v_{e}\right)$ ) with $1.0 \times 10^{20} \mathrm{PoT}$ at the JPARC synchrotron $(30 \mathrm{GeV})$. The same amount would be collected at the Fermilab Main Ring $(120 \mathrm{GeV})$ with $2.4 \times 10^{19}$ PoT or at the CERN SPS $(400 \mathrm{GeV})$ with $1.1 \times 10^{19}$ PoT. These scenarios correspond to 2-3 months of data taking in the present running performances for such proton drivers. Fig. 2 shows the energy distribution of $v_{e}^{C C}$ interactions for $10^{20}$ PoT in the $500 \mathrm{t}$ detector. The spectrum is well suited for the region of interest of DUNE. If the detector is placed off-axis it would cover the Hyper-Kamiokande region, at the expenses of a larger exposure.

Event rates. $0.5 \mathrm{kt}, 1.0 \mathrm{e}+20 \mathrm{pot}, \mathrm{L}=0.1 \mathrm{~km}$

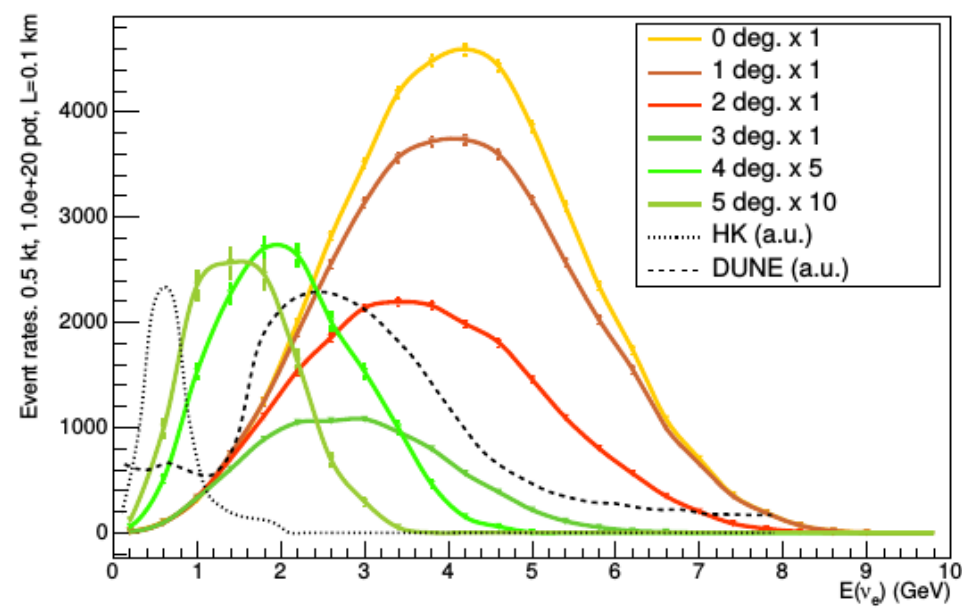

Figure 2: $v_{e}^{C C}$ events at the $v$-detector (500 tons) as a function of the neutrino energy. The rates are shown at different off-axis angles of the detector $\left(0^{\circ}-5^{\circ}\right)$. The events correspond to $1 \times 10^{20}$ PoT at the Fermilab Main Ring $(120 \mathrm{GeV})$ with the detector located $50 \mathrm{~m}$ after the beam dump $(0.1 \mathrm{~km}$ after the entrance of the decay tunnel). The dotted and dashed lines show the Hyper-K and DUNE neutrino spectrum, respectively.

\section{Design of the hadronic beamline}

The ENUBET secondary line has to focus and transport a collimated beam of sign and momentum selected pions and kaons with high efficiency, limited losses by decays and low beaminduced backgrounds. Furthermore the beam emittance at the entrance of the decay tunnel needs to be optimized in order to prevent undecayed secondaries or muons from pions from hitting the instrumented walls of the tunnel causing unmanageable pile-up effects.

The optimization of the line optics (dipole and quadrupole magnets) is performed with TRANSPORT [3], while the simulation of particle transport, collimation and reinteraction is accomplished by G4Beamline [4]. The dose assessment is done with FLUKA [5]. Studies are performed for different energies of the incoming protons $(30,120,400 \mathrm{GeV})$ and investigating two focusing systems: 
a conventional system based on a pulsed horn and a static one, implemented with large aperture dipoles/quadrupoles. An example of the latter is shown in Fig. 3.

The requirement for a local particle rate not exceeding $500 \mathrm{kHz} / \mathrm{cm}^{2}$, in order to limit pile-up effects in the positron tagger, suggests multi-Hz slow resonant proton extractions with $\mathscr{O}(\mathrm{ms})$ duration. On the other hand, extractions significantly longer than $10 \mathrm{~ms}$ are disfavoured if secondary focusing is achieved by magnetic horns. A static focusing solution could overcome these constraints, even if with a reduced secondary capture efficiency. It would allow a more conventional scheme with longer extractions, $\mathscr{O}(\mathrm{s})$, that is beneficial in terms of pile-up effects and that could ease the implementation of the time tagged configuration.

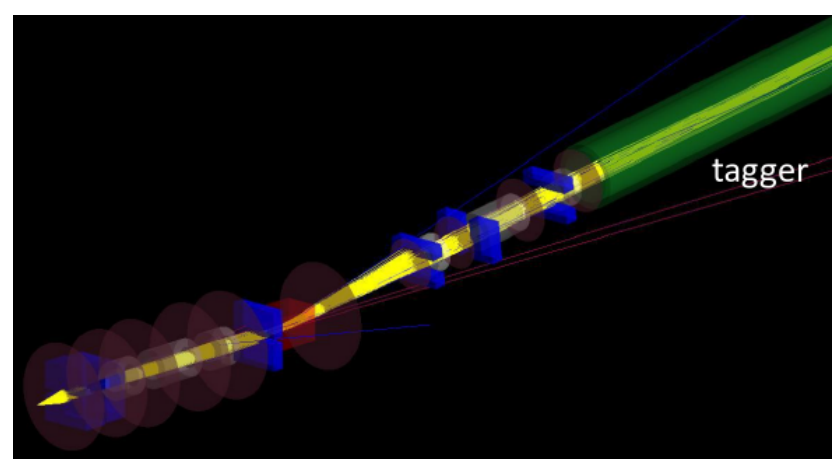

Figure 3: Layout of the hadronic beam line, as simulated in G4Beamline, based on an upstream quadrupole triplet, a bending dipole and a downstream quadrupole triplet. Charged particle trajectories are drawn in yellow, collimators in blue, the dipole in red, quadrupoles in grey, the positron tagger in green.

\section{The positron tagger}

The reference design of the positron tagger is based on calorimetric units (UCM) made of five, $15 \mathrm{~mm}$ thick, iron layers, interleaved with $5 \mathrm{~mm}$ thick plastic scintillator tiles, that instrument the two inner layers of the calorimeter. The total thickness of the UCM module $(10 \mathrm{~cm})$ corresponds to $4.3 X_{0}$ and its transverse size is $3 \times 3 \mathrm{~cm}^{2}$. The readout is performed in shashlik mode through nine WLS fibers coupled to silicon photomultipliers (Fig. 5, left). Six outer layers of shashlik modules with a longitudinal coarser readout (every $60 \mathrm{~cm}$, i.e. 2.6 interaction lengths) complete the calorimeter. They are used to measure the energy released by pions and muons, while e.m. showers will release their energy in the two innermost layers. The photon veto is made of doublets of $5 \mathrm{~mm}$ thick plastic scintillator pads with a surface of $3 \times 3 \mathrm{~cm}^{2}$, separated by a distance of $5 \mathrm{~mm}$. They are placed below the calorimeter every $7 \mathrm{~cm}$ and provide a full azimuthal coverage (Fig. 1, right).

A full GEANT4 [6] simulation of the instrumented decay tunnel has been setup to test its performance in terms of particle identification and optimize its design. In order to cope with pile-up effects at the expected particle rates ( $\sim 500 \mathrm{kHz} / \mathrm{cm}^{2}$ at the tagger inner surface), an event building algorithm to cluster correlated energy deposits based on their position and timing was developed. The e/ $\pi$ separation is then performed by a multivariate analysis based on a neural network that exploits differences in the energy deposition patterns of hadronic and electromagnetic showers. The rejection of $\pi^{0}$ related events is done using the information of photon veto scintillator tiles clustered in the event, requiring an energy deposition compatible with the passage of a m.i.p. [7]. 
As can be seen in Fig. 4 (left plot), the $\mathrm{K}_{e 3}$ selection efficiency, that amounts to $\sim 40 \%$ in ideal conditions and with a tunnel radius of $40 \mathrm{~cm}$, is degraded by pile-up effects to below 20\%. A mitigation is obtained by enlarging the tunnel at the expense of a reduced geometrical acceptance, with a final tagging efficiency of about $25 \%$ with the calorimeter at $1 \mathrm{~m}$ from the beam axis. In these conditions the purity of the selection amounts to $\sim 50 \%$. In fig. 4 (right plot) are shown the contributions from mis-identified events.
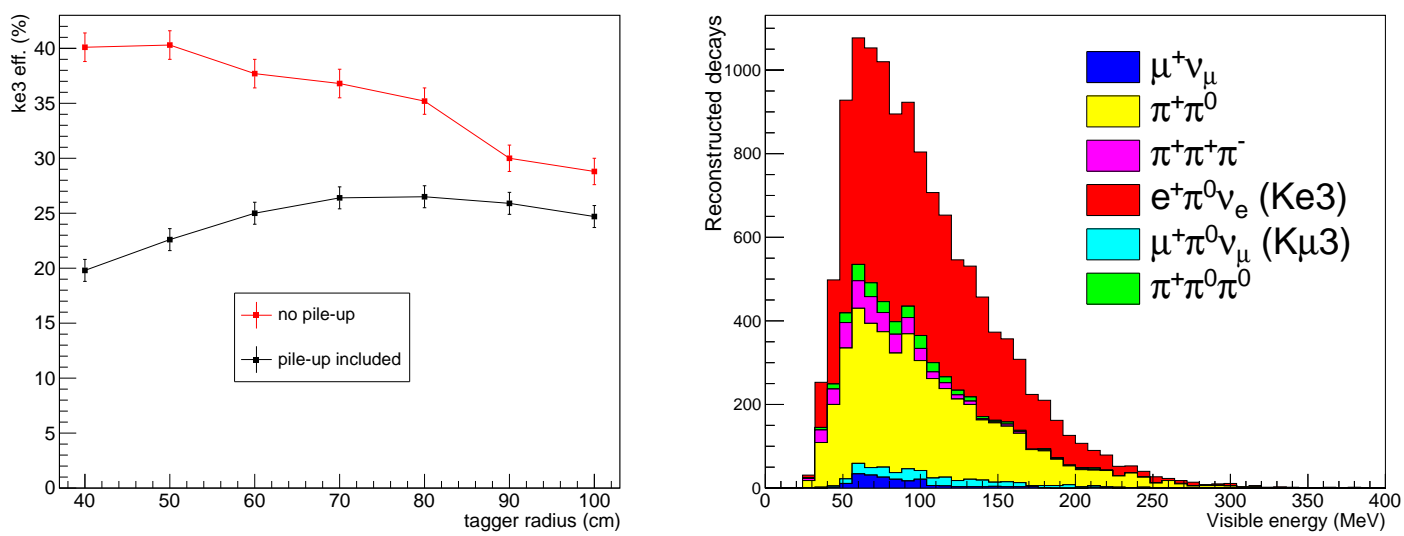

Figure 4: (Left) $\mathrm{K}_{e} 3$ selection efficiency as a function of the decay tunnel radius. In red are shown efficiencies when events are temporally separated. In black are reported the efficiencies when including pile-up effects at the nominal meson rate. (Right) Contribution of different kaon decay modes to all the events reconstructed as $\mathrm{K}_{e 3}$.

A larger radius allows for less stringent emittance requirements in the focusing line design. Furthermore it could be beneficial for the radiation hardness properties of the instrumentation. A FLUKA based simulation demonstrates that the integrated doses corresponding to $10^{4} v_{e}^{C C}$ events for $1 \mathrm{~m}$ radius amount to $<0.05 \mathrm{kGy}$ and $1.9 \times 10^{11} \mathrm{n} / \mathrm{cm}^{2}(1 \mathrm{MeV}$ equivalent), i.e. a factor $\sim 4$ less than for a $40 \mathrm{~cm}$ tunnel radius [8].

Recent investigations have shown that the positron tagging performances are almost unaffected when reducing the number of outer calorimetric layer from six to one, both in terms of efficiency and purity, thus allowing for a sensible reduction of readout channels with respect to the original design. Additional studies are ongoing to further optimize the tagger and the full picture is being updated taking into account the simulation of the transfer line in terms of beam related background and incoming particle rates, with the final goal of assessing the systematics to the neutrino flux due to the detector response.

\section{Detector prototyping and test beams activities}

An intense R\&D program, supported by test exposures to cosmic rays and charged particle $(\mathrm{e} / \pi / \mu)$ beams at the CERN-PS East Area Facility $[9,10]$, has led to the definition of the UCM reference design, as described in the previous section. The actual implementation, sketched also in Fig. 5 (left), has a readout based on nine, $1 \mathrm{~mm}$ diameter, WLS fibers running through the five iron-scintillator tiles, that are connected directly to $1 \mathrm{~mm}^{2}$ SiPMs. The fibers are inserted into 
a 3D printed mask located downstream of each UCM. Suitable grooves in the mask are used to host the SiPMs that are soldered on a printed circuit board. The coupling with the WLS fibers is done in air. Unlike conventional shashlik calorimeters, this scheme avoids the occurrence of large passive regions usually needed to bundle the fibers and route them to a common photo-sensor, thus improving the homogeneity in the longitudinal sampling.
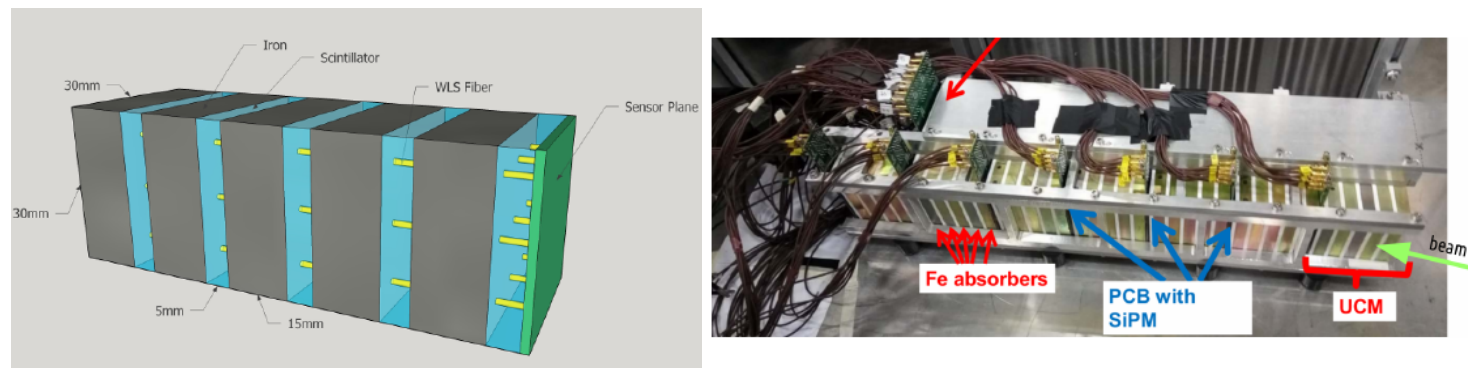

Figure 5: (Left) Layout of a UCM. (Right) Prototype exposed at CERN-PS T9 beamline in November 2016. The inner part of the calorimeter (bottom in the picture) is composed by 7 consecutive blocks of $4 \times 2$ UCM modules, coupled to PCBs for the readout of each UCM. The outer part (up in the picture) is a $60 \mathrm{~cm}$ long unique block acting as an energy catcher. The system was mounted on a tunable mechanical cradle to easily sample the incident angle of the particle beam.

The test beam exposures performed at the T9 beamline of the CERN-PS in July and November 2016, August and October 2017, were intended to investigate extensively a variety of technical solutions as scintillator material and thickness, WLS fiber types, SiPM-fiber coupling. Test aimed also at characterizing different prototypes in terms of energy resolution and linearity of response to electromagnetic showers, checking at the same time the viability of the UCM self-calibration with minimum ionizing particles. Furthermore they allowed to validate the capabilities of the GEANT4 simulation in reproducing the data distribution. In particular, during the November 2016 CERN test beam, a prototype of $56 \mathrm{UCMs}$ assembled in 7 longitudinal layers $\left(\sim 30 X_{0}\right)$ complemented by an outer module with a coarse reading was exposed to negatively charged particles at various incident angles (from 0 to $200 \mathrm{mrad}$ ) thus reproducing the working conditions of a sector of the final ENUBET tunnel (Fig. 5, right). The visible energy for a $0 \mathrm{mrad}$ tilt and for a $3 \mathrm{GeV}$ beam of pions, muons and electrons is shown for data and Monte Carlo in Fig. 6, left plot, while the right plot in the same figure illustrates the achieved energy resolution, well within the needs for an efficient e/ $\pi$ separation in ENUBET [11].

The reference detector option requires SiPMs embedded in the bulk of the calorimeters and, hence, exposed to radiation. Since SiPM are sensitive to neutron damage, a dedicated irradiation test has been performed at the CN irradiation facility at INFN-LNL (Legnaro) in summer 2017 [12]. SiPMs of different pixel size produced by FBK were irradiated up to $10^{12} \mathrm{n} / \mathrm{cm}^{2}(1 \mathrm{MeV}$-eq.) and their response in the UCM were tested at the CERN East Area facility.

Non conventional solution like polysiloxane based scintillator or non-shashlik readout are being investigated in the already accomplished test beam exposures or in the ones foreseen for 2018.

\section{Acknowledgements}

This project has received funding from the European Union's Horizon 2020 Research and 

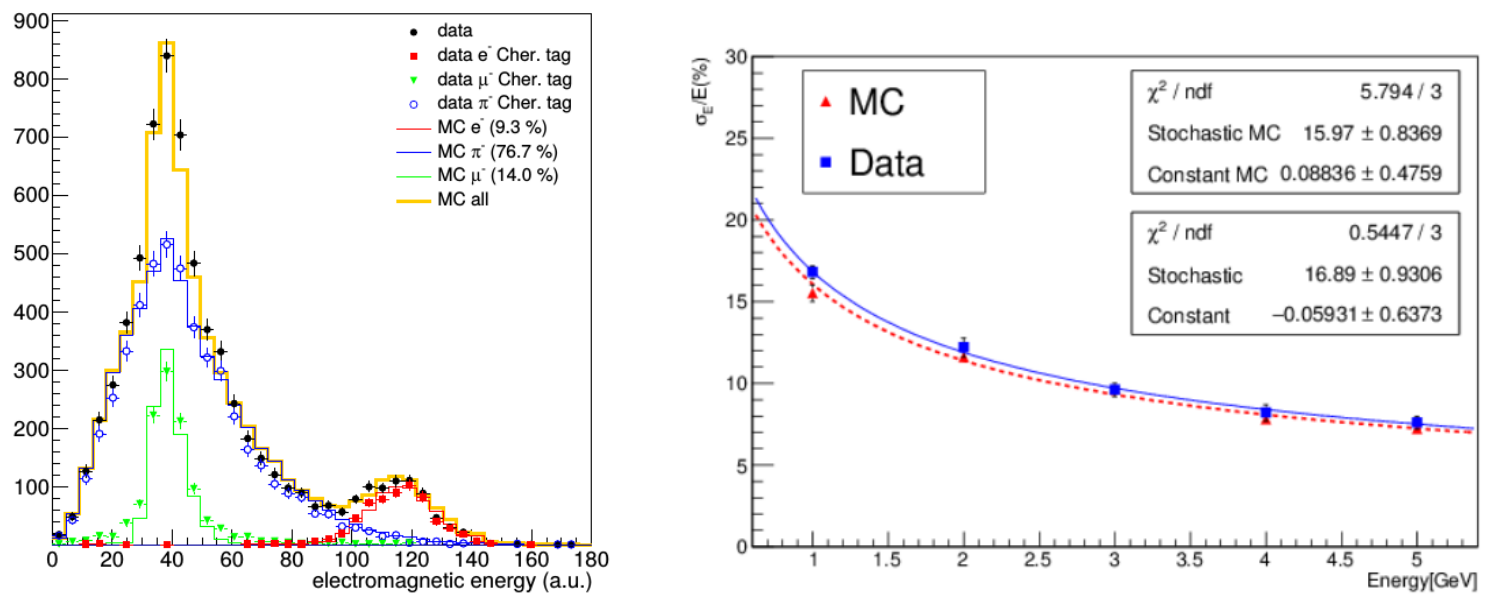

Figure 6: (Left) Distribution of the energy deposited in the scintillator of the calorimeter exposed in November 2016 to $\mathrm{e}^{-} / \pi^{-} / \mu^{-}$in the T9 beamline. The particle tagging in data is performed with two Cherenkov threshold chambers equipping the line, and the measured beam composition is reported in parenthesis. (Right) Energy resolutions for electrons, fitted with the usual parametrization $\frac{\sigma(E)}{E}=\frac{s}{\sqrt{E}} \oplus c$.

Innovation programme under Grant Agreement no. 654168 and no. 681647.

\section{References}

[1] A. Longhin, L. Ludovici and F. Terranova, Eur. Phys. J. C 75, 155 (2015).

[2] A. Berra et al. [ENUBET Coll.], CERN-SPSC-2016-036; SPSC-EOI-014.

[3] K.L. Brown et al., TRANSPORT, A computer program for designing charged particle beam transport systems, May 1983, http://lss.fnal.gov/archive/nal/fermilab-nal-091.pdf.

[4] http://www.muonsinternal.com/muons3/G4beamline

[5] The FLUKA Code: Developments and Challenges for High Energy and Medical Applications, T.T. Bühlen, F. Cerutti, M.P.W. Chin, A. Fassò, A. Ferrari, P.G. Ortega, A. Mairani, P.R. Sala, G. Smirnov and V. Vlachoudis, Nuclear Data Sheets 120, 211-214 (2014).

FLUKA: a multi-particle transport code, A. Ferrari, P.R. Sala, A. Fassò, and J. Ranft, CERN-2005-10 (2005), INFN/TC_05/11, SLAC-R-773.

[6] S. Agostinelli et al., [GEANT4 Coll.], Nucl. Instrum. Meth. A 506, 250 (2003).

[7] A. Meregaglia et al., JINST 11 no.12, C12040 (2016).

F. Pupilli et al., Positron identification in the ENUBET instrumented decay tunnel, to appear on the Neutrino Telescopes 2017 conference proceedings.

[8] A. Longhin et al., High precision flux measurements in conventional neutrino beams: the ENUBET project, to appear on the Neutrino Telescopes 2017 conference proceedings.

[9] A. Berra et al., Nucl. Instrum. Meth. A 830, 345 (2016).

[10] A. Berra et al., IEEE Trans.Nucl.Sci. 64 no.4, 1056 (2017).

[11] A. Berra et al., JINST 13 no.01, P01028 (2018).

[12] V. Mascagna, Talk at the 8th International Conference on New Development in Photodetectors (NDIP 2017), Tours, France, 3-7 July 2017. 